\title{
Assessment of macular function by multifocal electroretinogram before and after macular hole
}

\section{surgery}

\author{
Ying-Jie Si, Shoji Kishi, Koji Aoyagi
}

\begin{abstract}
Aim-To evaluate macular function before and after successful surgical closure of idiopathic macular holes using multifocal electroretinogram (ERG).

Methods-40 patients (40 eyes) with idiopathic macular holes were examined using multifocal ERG both before and after vitreous surgery. The postoperative period was from 1 to 12 months.

Results-Preoperatively, the electrical retinal response densities in the foveal and the perifoveal area were apparently decreased. After a mean postoperative period of 3-6 months, the foveal and perifoveal area electrical retinal response densities improved to two to four times the preoperative level and the improvement continued to 1 year after surgery.

Conclusion-In macular holes, the decrease in retinal electrophysiological response was not limited to the fovea but involved an area of the perifovea of $\mathbf{1 . 6}$ disc diameters. The electrical retinal response density of these areas gradually improved after macular hole closure.

(Br f Ophthalmol 1999;83:420-424)
\end{abstract}

Idiopathic macular holes are treatable with vitrectomy. After successful hole closure, most patients gain two or more lines of visual acuity until 6-12 months postoperatively, and resolution of metamorphopsia occurs. The mechanism by which visual function improves is not understood. Visual function before and after macular hole surgery generally is assessed by visual acuity measurement. ${ }^{1-6}$ However, the visual acuity level represents only a part of the impaired visual function resulting from macular hole development, which includes metamorphopsia, blurred vision, and scotoma. There have been few methods to evaluate these subjective symptoms. Some researchers used objective methods such as focal electroretinogram (ERG) to evaluate macular function, ${ }^{7-10}$ which reflects the different spatial distributions of the retinal receptors and supporting tissues. However, this method requires prolonged recording times and varying signal to noise ratios. Because focal ERG was designed to assess the macular area within 10 degrees, it cannot evaluate the electrophysiological response in the perimacular area. Multifocal ERG, introduced by Sutter et $a l,{ }^{11}{ }^{12}$ resolves these limitations, because multiple retinal loca- tions can be tested simultaneously, and allows functional mapping of the retina.

\section{Patients and methods}

Forty eyes of 40 patients diagnosed with idiopathic macular holes in stages 2 to 4 (three, 34 , and three eyes, respectively), according to Gass's classification, ${ }^{13}{ }^{14}$ were examined by multifocal ERG before and after vitrectomy. The ages of the patients ranged from 48 to 78 years (average 64 years). The macular holes were successfully closed in all 40 eyes. All the patients were examined and diagnosed by the same doctor (SK) before and after surgery and those who had apparent cataract was excluded from our study in order to avoid the cataract influence on our multifocal ERG study. Multifocal ERG were recorded preoperatively and 3 months, 6 months, and 1 year after surgery. A visual evoked response imaging system (VERIS III, Tomey Corp, Nagoya, Japan) was used for the ERG recording. The multifocal ERG stimulus matrix consisted of 103 hexagons that scale concentrically and covered the central 50 degrees of the fundus area (Fig 1A). Each hexagon alternated between black and white in binary $\mathrm{m}$-sequences at the rate of 75 $\mathrm{Hz}$. The range of luminance levels is $5-200$ $\mathrm{cd} / \mathrm{m}^{2}$. The central hexagon (area 1, foveal area) corresponded to approximately 2.8 degrees of the fundus area. We used the cuneiform indicator rather than the pointer for fixation because patients had a central scotoma. A Burian-Allen bipolar contact lens electrode was used for signal derivation. A ground electrode was attached to the earlobe. The pupil of one eye was dilated with $0.5 \%$ tropicamide and $0.5 \%$ phenylephrine hydrochloride, and the fellow eye was occluded. The net recording time for each eye was 4 minutes; the entire procedure was divided into eight 30 second segments. The signal were amplified (100 000) and bandpass filtered $(10-300 \mathrm{~Hz})$. Three dimensional topography (Fig 1B) represented the retinal response density (amplitude per retinal area, $\mathrm{nV} / \mathrm{deg}^{2}$ ). The recording procedure was repeated if there were spurious potentials from eye blinks or ocular movement were recorded.

\section{Results}

The mean preoperative retinal response densities in 40 eyes with macular holes (three eyes with stage 2, 34 with stage 3 , and three with stage 4) examined were 3.77 (SD 2.03) $\mathrm{nV} / \mathrm{deg}^{2}$ in area 1 (foveal area of 2.8 degrees) and $3.82(2.58) \mathrm{nV} / \mathrm{deg}^{2}$ in area 2 (perifoveal
Accepted for publication 22 September 1998 
area of 3-9 degrees from the centre). At the examination performed 3 months postoperatively, the mean retinal response densities improved to $6.99(2.69) \mathrm{nV} / \mathrm{deg}^{2}$ in area $1 ; 5.01$ (2.54) $\mathrm{nV} / \mathrm{deg}^{2}$ in area 2 . Six months after surgery the mean retinal response density further improved to $8.27(2.69) \mathrm{nV} / \mathrm{deg}^{2}$ in area 1 while the mean retinal response density in area 2 was at the same level $\left(5.16(2.38) \mathrm{nV} / \mathrm{deg}^{2}\right)$. Figure 2 shows the relation of the retinal response densities in area 1 before and 6 months after surgery in the 40 eyes ( $\mathrm{rp}=0.57$, $\mathrm{p}<0.001)$. The retinal response density improved even in three eyes with unchanged visual acuity. Although a positive correlation was seen between the postoperative retinal response density and the visual acuity $(\mathrm{r} p=0.46, \mathrm{p}<0.01)$, the retinal response density varied at wide range in eyes with the same visual acuity (Fig 3).

The retinal response density gradually increased until 1 year postoperatively in six of the 40 patients who were examined by the multifocal ERG 12 months after vitrectomy. The mean central postoperative retinal response density (area 1) was 8.74 (2.45) $\mathrm{nV} / \mathrm{deg}^{2}$.

CASE 1

A 75 year old man with a stage 3 macular hole in his left eye had a preoperative visual acuity
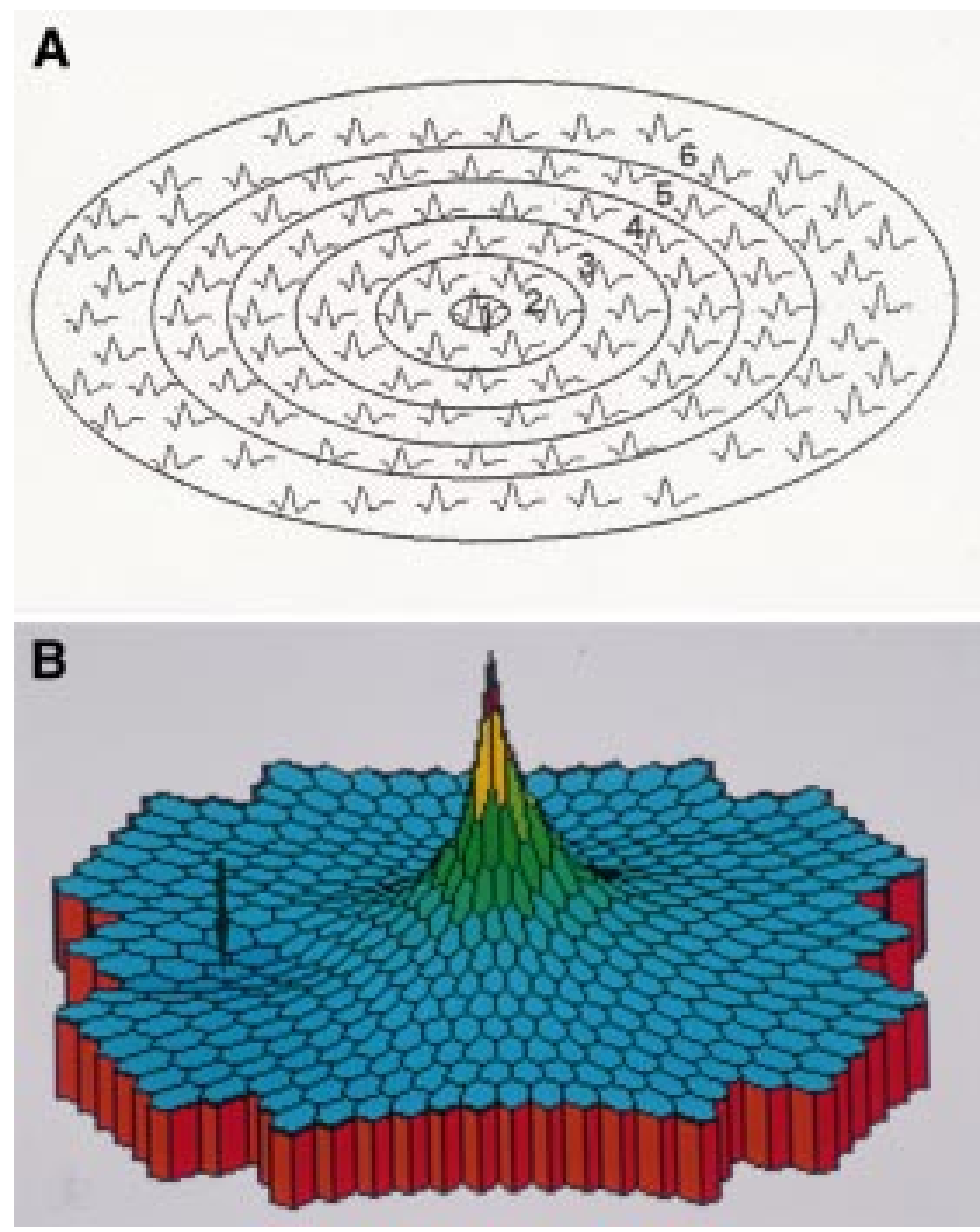

Figure 1 Schema of the stimulus matrix of multifocal ERG $(A)$, and three dimensional scalar plots of ERG responses obtained from the left eye of a normal subject $(B)$. There is a sharp peak at the foveal area and slight depression at the optic disc (arrow). level of 0.1 . The topography of the retinal response density was markedly depressed in the macular area (Fig 4A, B). The retinal response density of area 1 was $4.14 \mathrm{nV} / \mathrm{deg}^{2}$. A multifocal ERG was repeated at 1,3 , and 6 months postoperatively. The retinal response density of area 1 improved to approximately $5.78,7.76,11.91 \mathrm{nV} / \mathrm{deg}^{2}$ accompanied by a visual acuity improvement to $0.2,0.3$, and 0.5 , respectively. The postoperative changes of multifocal ERG topography are seen in Figure $4(\mathrm{C}, \mathrm{D}$ and $\mathrm{E}, \mathrm{F})$.

\section{CASE 2}

A 62 year old man with a stage 3 macular hole in his right eye underwent a preoperative multifocal ERG, the topography of which showed a marked central depression of approximately 8 degrees both in the hole and the surrounding area. The central retinal response density was $3.17 \mathrm{nV} / \mathrm{deg}^{2}$ preoperatively and improved to $9.12 \mathrm{nV} / \mathrm{deg}^{2} 6$ months postoperatively. The visual acuity improved from 0.1 to 0.4 (Fig 5).

\section{Discussion}

The results of multifocal ERGs recorded preoperatively in 40 eyes showed marked decreases in retinal response densities that were not only limited to the fovea but also involved the perifoveal area. We previously observed the morphological features of macular holes using scanning laser ophthalmoscopy. ${ }^{15}$ Henle's fibre layer was elevated around the macular holes, forming radiating striae. They were seen in approximately 10 degrees of the perifoveal area in full thickness holes. The findings of optical coherence tomography indicated that the perifoveal radiating striae represent intraretinal splitting or retinoschisis. ${ }^{16}$ Decreased retinal response density in the perifoveal area (area 2) seems to reflect perifoveal retinoschisis.

The improvements in the retinal response density in the foveal area (area 1) appeared to reflect the closure of the macular holes with

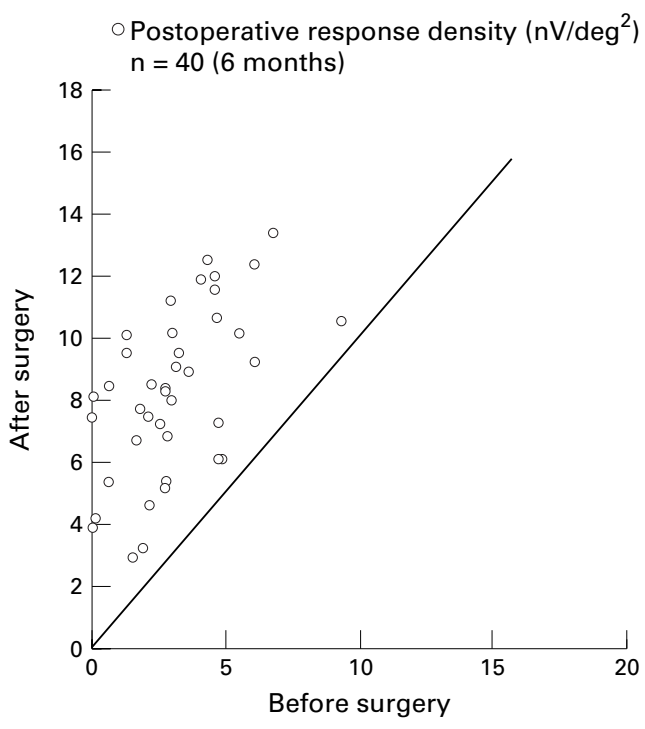

Figure 2 Relation between preoperative and postoperative retinal response density 6 months after surgery. Retinal response density increased in all eyes $(p<0.001)$. 
Figure 3 Association between visual acuity and the retinal response density 6 months after surgery. A positive correlation between response density and visual acuity is seen $(p<0.01)$. Retinal response density varied in eyes with the same visual acuity.

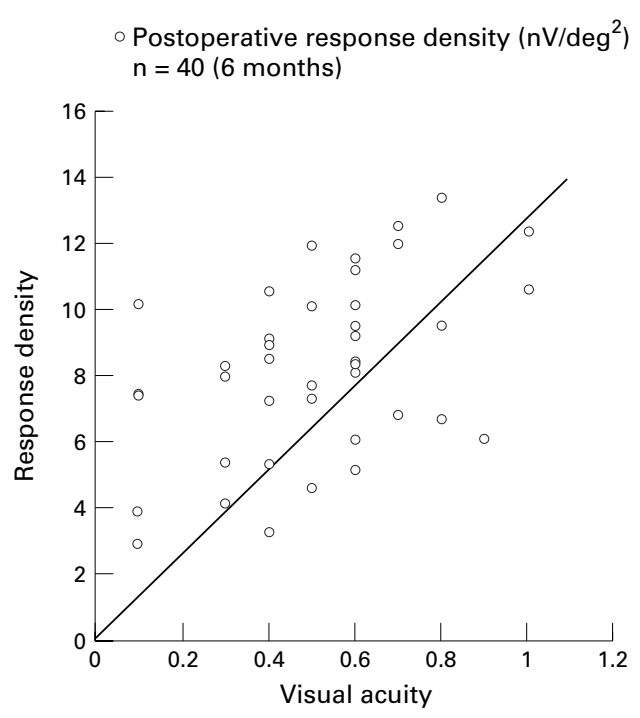

proliferated glial cells and centripetal displacement of surrounding photoreceptor cells. ${ }^{17}{ }^{18}$ Resolution of the central scotoma seems to be attributed to anatomical repair in area 1 . The radiating striae of the Henle's fibre layer disappeared following macular hole closure. ${ }^{15}$ The improvement of retinal response density in area 2 along with resolution of metamorphopsia is presumably achieved by reattachment of perifoveal retinoschisis. The improvement of the retinal response density continued to 12 months postoperatively. The increases in the mean postoperative retinal response density in the 40 study eyes in area 1 indicate that the intraretinal neural network was restored with time after the closure of the macular holes.

The origin of the electrophysiological responses in the multifocal ERG remains controversial. Recently, some researchers studied the effect of filter bandwidth on the multi-
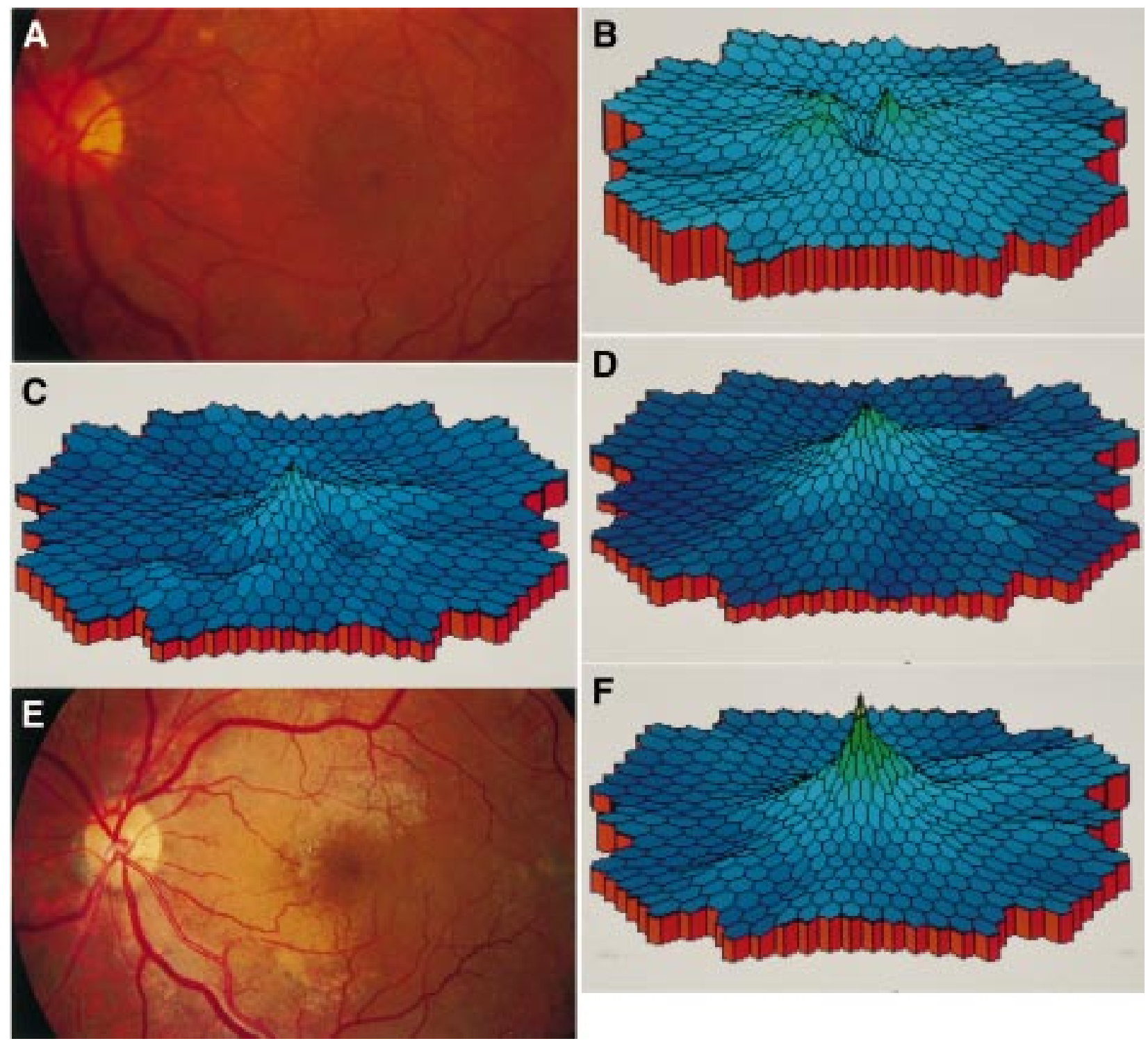

Figure 4 Multifocal ERG of a 75 year old man with a stage 3 macular hole (case 1, left eye) before and after surgery. Preoperative fundus photograph (A) and multifocal ERG topography (B). Foveal and perifoveal area (areas 1 and 2) showed crater like depression. Multifocal ERG recorded at 1 month (C) and 3 months (D) postoperatively. The peak recovered in the macular area. Fundus photograph (E) and multifocal ERG topography (F) 6 months postoperatively. 

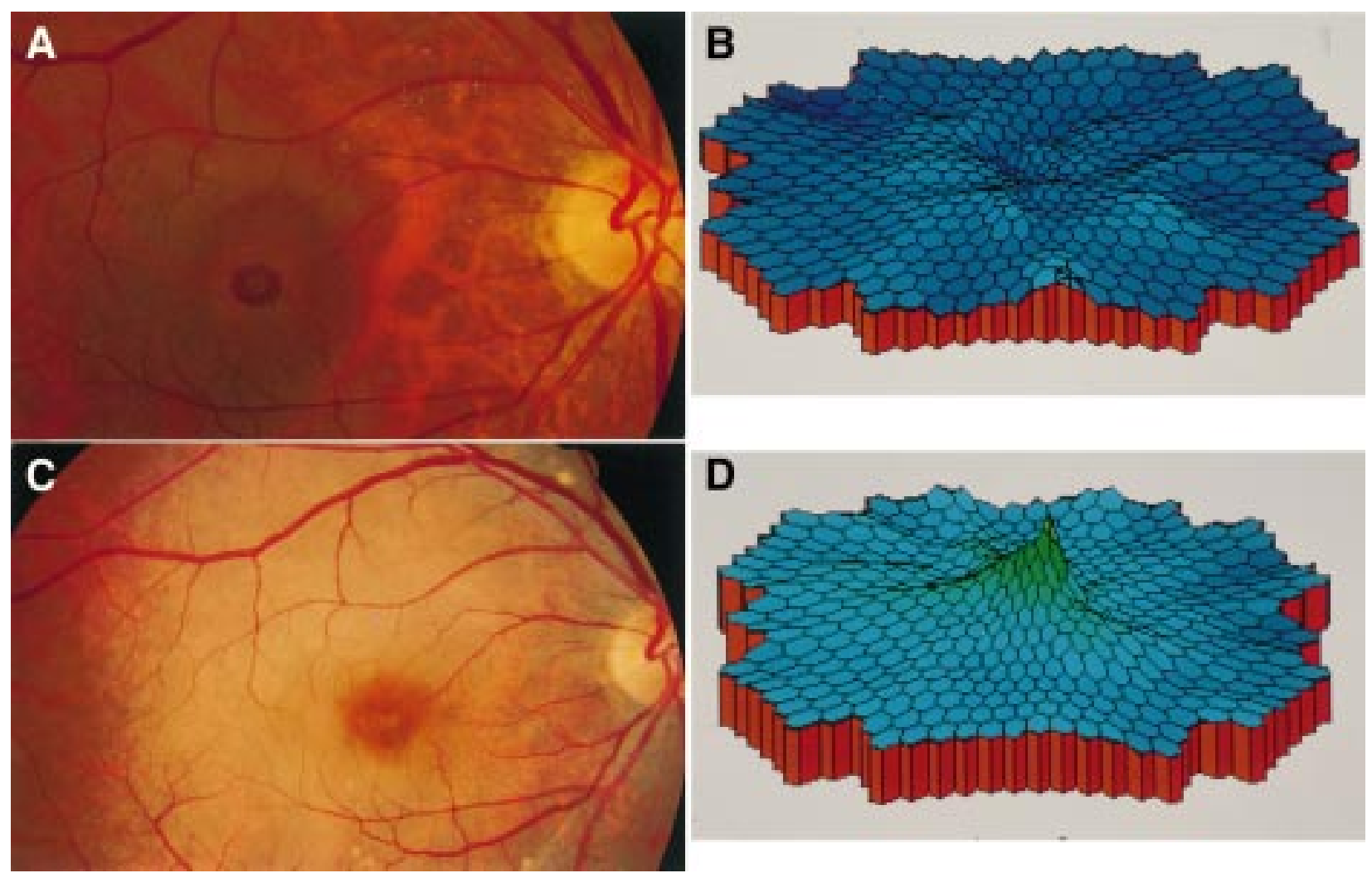

Figure 5 Fundus photograph and multifocal ERG of a 62 year old man with a stage 3 macular hole (case 2, right eye) before and after surgery. (A) Fundus photograph preoperatively. Macular hole is larger than that of the patient in case 1. (B) Multifocal ERG topography showed more widespread crater-like depression in macular area than that in case 1. Fundus photograph (C) and multifocal ERG topography (D) 6 months postoperatively. Macular hole had closed. The peak of the retinal response density was restored in macular area in the topography of multifocal ERG.

focal ERG. ${ }^{19}$ Other researchers recorded multifocal ERG in normal eyes and eyes with diabetic retinopathy. ${ }^{20-22}$ A multifocal ERG study in eyes with branch retinal artery occlusion demonstrated decreased electrophysiological responses in the ischaemic area, ${ }^{23}$ suggesting that the multifocal ERG reflects not only the electrophysiological responses of cones but also that of the inner retinal layer including bipolar and Mueller's cells. In eyes with macular holes, a decreased retinal response density seems to reflect the loss of photoreceptors in the fovea and the retinoschisis in the perifoveal area. Multifocal ERG is a useful method to evaluate retinal function in macular holes before and after surgery.

In full thickness macular holes, electrophysiological dysfunction was not limited to the fovea but involves its surrounding area. Reparation of the intraretinal neural network seems to continue up to 1 year after surgical closure of macular holes.

Presented in part on 14 May at the 1997 annual meeting of the Association for Research in Vision and Ophthalmology, Fort Lauderdale, Florida, USA.

1 Sjaarda RN, Frank DA, Glaser BM, et al. Resolution of an absolute scotoma and improvement of relative scotoma after successful macular hole surgery. Am f Ophthalmol 1993;116:129-39.

2 Fish GE, Birch DG. The focal electroretinogram in the clinical assessment of macular disease. Ophthalmology 1989;96:109-14.

3 Sjaarda RN, Frank DA, Glaser BM, et al. Assessment of vision in idiopathic macular hole with macular microperimetry using the scanning laser ophthalmoscope. Ophthalmology 1993;100:1513-18.
4 Nussenblatt RN, Kaufman SC, Palestine AG, et al. Macular thickening and visual acuity. Ophthalmology 1987;94: 1134-9.

5 Smiddy WE, Thomley ML, Knighton RW, et al. Use of the potential acuity meter and laser interferometer to predict visual acuity after macular hole surgery. Retina 1994;14: 205-9.

6 Fish GE, Birch DG, Fuller DG, et al. A comparison of visual function tests in eyes with maculopathy. Ophthalmology 1986;93:1177-82.

7 David GB, Bradley FJ, Gary EF. The focal electroretinogram in fellow eye of patients with idiopathic macular holes. Arch Ophthalmol 1988;106:1558-63.

8 Acosta FA, Lashkari K, Reynaud X, et al. Characterization of functional changes in macular holes and cysts. Ophthalmology 1991;98:1820-23.

9 Seiple WH, Seigel IM, Carr RE, et al. Evaluating macular function using the focal ERG. Invest Ophthalmol Vis Sci 1986;27:1123-30.

10 Fish GE, Birch DG. The focal electroretinogram in the clinical assessment of macular disease. Ophthalmology 1989;96:109-14.

11 Sutter EE, Tran D. The field topography of ERG components in man. 1. The photopic luminance response. Vision Res 1992;32:433-66.

$12 \mathrm{Wu}$ S, Sutter EE. A topographic study of oscillatory potentials in man. Visual Neurosci 1995;12: 1013-25.

13 Gass JD. Idiopathic macular hole. Its early stages and pathogenesis. Arch Ophthalmol 1988;106:629-39.

14 Johnson RN, Gass JD. Idiopathic macular holes. Observations, stages of formation, and implications for surgical intervention. Ophthalmology 1995;95:91724.

15 Kishi S, Kamei Y, Shimizu K. Tractional elevation of Henle's fiber layer in idiopathic macular holes. Am F Ophthalmol 1995;120:486-96

16 Hee MR, Puliafito CA, Wong C, et al. Optical coherence tomography of macular holes. Ophthalmology 1995;102: $748-56$

17 Funata NE, Wendel RT, Zenaida de la Crus, et al. Clinicopathologic study of bilateral macular holes treated with pars plana vitrectomy and gas tamponade. Retina 1992;12: 289-98.

18 Madreperla SA, Geiger GL, Funata M, et al. Clinicopathologic correlation of a macular hole treated by cortical vitreous peeling and gas tamponade. Ophthalmology 1994;101: 682-6.

19 Keating D, Parks S, Evans AL, et al. The effect of filter bandwidth on the multifocal electroretinogram. Doc Ophthalmol 1997;92:291-300. 
20 Parks S, Keating D, Williamson TH, et al. Functional imaging of the retina using the multifocal imaging of the retina using the multifocal
electroretinogram: a control study. $\mathrm{Br} F$ Ophthalmol 1996;80:831-4

21 Kondo M, Miyake Y, Horiguchi $M$, et al. Normal values of retinal response densities in multifocal electroretinogram. $\mathcal{F}$ fpn Ophthalmol Soc 1996;100:810-16
22 Palmowsky AM, Sutter EE, Bearse MA Jr, et al. Mapping of retinal function in diabetic retinopathy using the multifocal electroretinogram. Invest Ophthalmol Vis Sci 1997;38. 2586-96

23 Kondo M, Miyake Y, Horiguti M, et al. Clinical assessment of multifocal electroretinogram. Invest Ophthalmol Vis Sci 1995;36:2146-56. 